\title{
Light Fidelity (Li-Fi) Is it Possible to Replace Wi-Fi?
}

\author{
Rabie A. Ramadan \\ Computer Engineering Department \\ Cairo University \\ Cairo, Egypt, on leave at Hail University, KSA \\ rabie@rabieramadan.org
}

\author{
Ahmed Y. Khedr \\ Systems and Computer Department \\ Al-Azhar University, Cairo, Egypt, on leave at Hail \\ University , KSA \\ a.khedr@uoh.edu.sa
}

\begin{abstract}
-imagine you are walking in your company or a hospital and instead of trying to connect to an access point to reach the internet or downloading a file, the data is coming to you through the hospital or your company lights. Also, imaging that the data transfer rate is increased multiple times. Isn't great to have such technology? It seems that the dream comes true with the appearance of $\mathrm{Li}-\mathrm{Fi}$ technology. In this paper, we will overview the concept of $\mathrm{Li}-\mathrm{Fi}$, applications, its advantages, and the suitable types of modulations. By the end of this research, some of the disadvantages and open problems are presented.
\end{abstract}

Keywords-Li-Fi;Light Fedality ; New Technology; Light Modulation; Smart Environment .

\section{INTRODUCTION}

The idea behind Wi-Fi is to connect your devices together through wirelessly or getting the Internet broadband to your computer/device without wires. The Wi-Fi system is based on two parts which are the transmitter and receiver. The transmitter converts the data coming from the Internet into Radio signals that is converted back into data using the receiver.

Wi-Fi first released to the public in 1997 when IEEE 802.11 for Wireless Local Area Network (WLAN) standard was published. This standard allows data transfer with two megabits per second. Starting from 1999, there was a router as a prototype for Wi-Fi technology. The new technology advanced more by utilizing two different frequencies which are $2.4 \mathrm{GHz}(802.11 \mathrm{~b})$ and $5 \mathrm{GHz}$ (802.11a).

The year of 1999 had witnessed the birth of the term Multiple Input Multiple Output (MIMO) to describe the usage of multiple antennas for multiple data streams according to IEEE802.11n standard. In 2012, new standard has been released to achieve $5 \mathrm{Ghz}$ data transmission through Wi-Fi. The new standard allows the support of more antennas and greater bandwidth. The idea of increasing the data transfer speed is done through beamforming concept.

Wi-Fi typically consists of Access Points (APs) and one or more clients. The access point is considered as the gate to the service provider. The AP broadcasts a beacon message every $100 \mathrm{~ms}$ contains the Service Set identifier and network name (SSID). A client who receives this message has the opportunity to join the network through this AP or not. The Signal to Noise Ratio (SNR) may affect the client decision in joining one of the APs in its range.
The advantages of the Wi-Fi could be summarized as follows:

- Due to the unlicensed radio spectrum that Wi-Fi uses, there is no regulatory approval. So, it can be deployed anywhere.

- No cables are required to be deployed or even there is no need for cables.

- Many of the vendors nowadays producing access points and client interfaces with the required standards; so anyone can decide to have a Wi-Fi network at his/her place with low cost.

- Rooming is one of the main advantages of the Wi-Fi where a cellular device or laptops for example can join a hotspot network anywhere.

- Security becomes a major advantage to the Wi-Fi networks where different encryption techniques are now supported.

In 2012, new technology has been announced by the German physicist-Harald Haas [4]. He showed a prototype to a system at TED that can send and receive data through LED bulbs and LED detectors. He called this system Li-Fi. The idea attracted some of the researchers to investigate the validity of the concept. Nevertheless, we list some the current commercial products as well the startups.

Through this paper, we will go through the Li-Fi concept in some details. In addition, some of the new modulation techniques are proposed for this purpose. Moreover, we will discuss the issues and advantages of the Li-Fi technology.

The following section explains the main idea behind the Li-Fi technology; section III explains the proposed modulation techniques to serve the new concept of the data transmission. The commercial products and the new startups are presented in section IV. The discussion of the new technology is presented in section $\mathrm{V}$.

\section{LI-FI TECHNOLOGY}

Haas in 2011 took the stage in TED explaining idea behind the data transfer through the light. He demonstrated a simple prototype to the system and announced the success of the first prototype of Light Fidelity (Li-Fi) technology. Li-Fi uses the light as a media of data transfer. The light is the gift to the 
human and it is available everywhere; therefore the success of the Li-Fi will be a new revolution to the science of the data transfer. A new dimension will be added to the wireless data technology.

The main concept of $\mathrm{Li}-\mathrm{Fi}$ is to transfer the data through sending light through Light Emitting Diode (LED) Bulbs as shown in Figure 1.

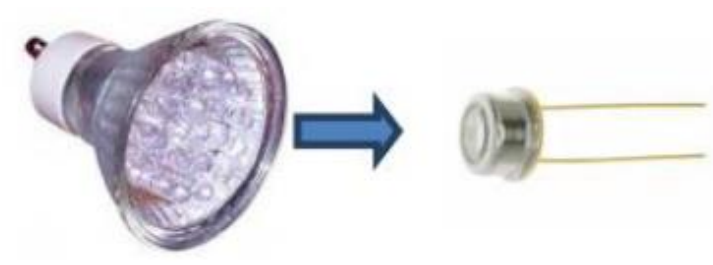

Fig. 1. Main concept of Li-FI technology

The transmission rate of the data can control the intensity of the bulb where the light intensity can vary more than the human eye can realize. For simultaneous data transmission, data is encoded on the intensity of light to control the data variation and does not affect the brightness of the light [4]. The transmitter is not limited to LEDs but could be any kind of light emitters could be a transmitter to the data; however, selecting one source of light could be better than others. Based on that the data transfer rate ranges from $40 \mathrm{Mbit} / \mathrm{s}$ to 100 Mbit/s. However, in 2010, Siemens announced that the Li-Fi data transmission rate could be $500 \mathrm{Mbit} / \mathrm{s}$. in fact, UK researchers [6] claimed that they can reach 10 Gbps using micro-LED light bulb to transmit $3.5 \mathrm{Gbps}$ via each of the three primary colors - red, green, blue - that make up white light.

At the receiver side, the light detectors are used to receive the sent data as shown in Figure 1. A simplified receiver of Li-Fi could be photodiodes in which it turns the light into electrical pulses. However, some other ideas such as using cameras to detect the light flickers could be also possible.

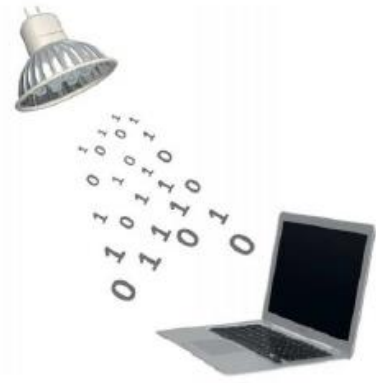

Fig. 2. Li-Fi main idea

The simplicity of the Li-Fi is that turning the LED on produces logic 1 and turning it off produces logic 0 as shown in Figure 2. In fact, the typical light bulb flickers 20,000 times a second while Fundan University researchers were able to flicker the light bulb billions of times per second.
A simplified block diagram is depicted in Figure 3. As can be seen in the Figure, the LEDs are connected to a network interface module that is directly connected to the Internet or Wireless Local Area Network (WLAN). To control the LEDs intensity a brightness control as a driving circuit is designed for this purpose. It is worth mentioning that the visible light wavelength ranges between 400 to $700 \mathrm{~nm}$. At the receiving side, there are an optical concentrator/detector, photodiode, and amplifier. The receiver is directly connected to the communication device.

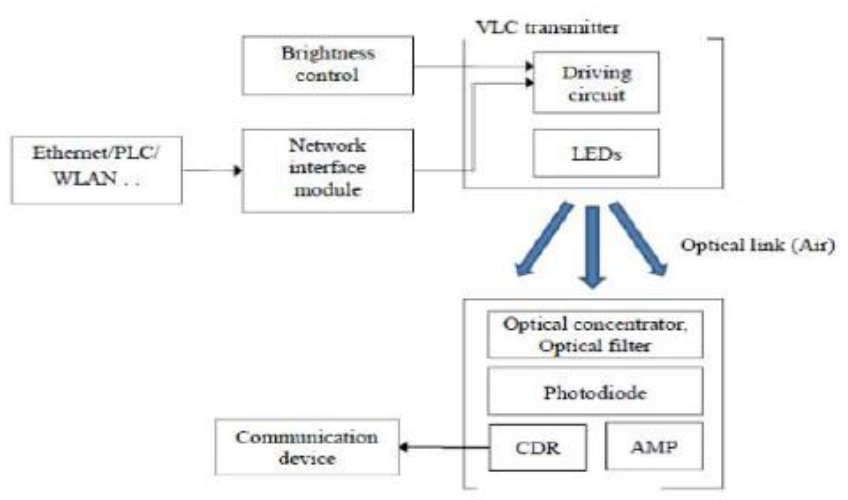

Fig. 3. Simplified block diagram of Li-Fi [9]

\section{FUTURE LI-FI APPLICATIONS}

In this section, some of the possible applications to $\mathrm{Li}-\mathrm{Fi}$ are presented. Some of these future applications are Smart Lighting, Hospital and Healthcare, Vehicles and Transportation, Aviation, Underwater Communications, and Indoor Positioning.

Smart Lighting: with any indoor and outdoor lights, the Internet service can be provided. Imagine you are walking in the street and you are connected to the street lamps with high speed and bandwidth providing you with the information you need.

Hospital and Healthcare: one of the effective applications of Li-Fi is providing the data transfer as well as patient's monitoring through the hospital lamps. In fact, the Li-Fi will be a clean media for data transfer that does not interfere with any of the medical equipments.

Vehicles and Transportation: it will be a great pleasure to drivers and travelers to enjoy the Internet and well entertainment through vehicles as well as buses, and trains lights. Not only that, it will beneficial in providing traffic and localization information. Vehicle to vehicle communication through the vehicles" front and rare lights to avoid accidents. Vehicles can also talk to the roadside units for safety and nonsafety information.

Aviation: passengers' cabins are full of LEDs and lamps. These LEDs can provide the entrainment services to the travelers in an easy way. In fact, we will not hear the "turn off your mobile devices" warning anymore. No interference with the aviation system will occur. 
Underwater Communications: there are many current problems with the underwater communication. In fact, the acoustic communication uses low bandwidth and affects the marine life. Li-Fi will be the best solution for such transmission media, safe and effective.

Indoor Positioning: localization techniques could be also utilized through lights. However, this time was very nature and safe technology.

\section{LI-FI MODULATION TECHNIQUES}

There are many modulation techniques that have been designed specifically for Wi-Fi. However and unfortunately, such techniques will not be suit for $\mathrm{Li}-\mathrm{Fi}$. Since all of $\mathrm{Wi}-\mathrm{Fi}$ modulation techniques depend mainly on preparing the data to be transferred through air interface through Radio Frequencies (RFs). In fact, the carrier signal in $\mathrm{Wi}-\mathrm{Fi}$ is the radio frequency while in Li-Fi is light pulses sent out in short intervals. The following are the proposed modulation techniques for $\mathrm{Li}-\mathrm{Fi}$.

Pulse-Position Modulation (PPM) is one of the modulation techniques in which $\mathrm{M}$ message bits are encoded by transmitting a single pulse in one of $2 \mathrm{M}$ possible required time-shifts. The pulses are repeated every $\mathrm{T}$ seconds in which $\mathrm{M} / \mathrm{T}$ bits are transmitted per second. All pulses are having the same width and amplitude. The delay between the pulses can be changeable as can be seen in Figure 4.

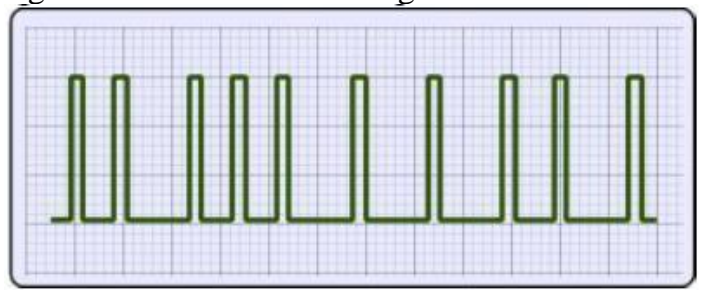

Fig. 4. Pulse position modulation example [3]

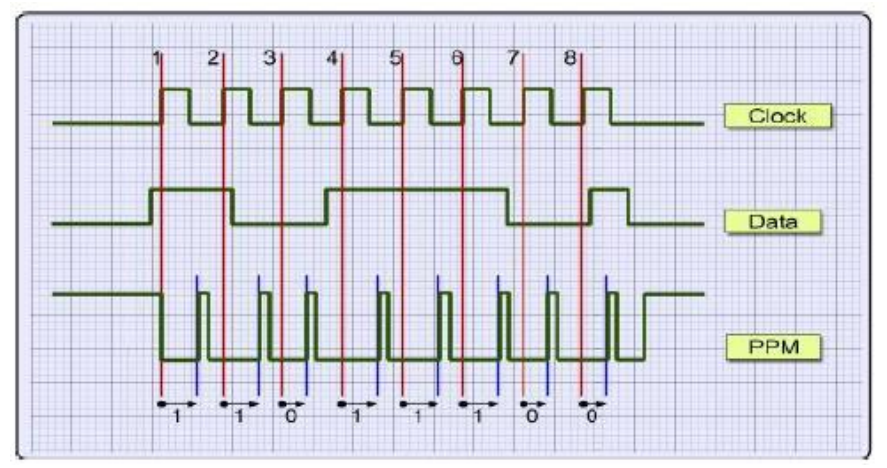

Fig. 5. Another example of the PPM [3]

Figure 5 is another example on the PPM modulation. A delay of $1.2 \mathrm{mSec}$ represents digital 0 , and a delay of $1.8 \mathrm{mSec}$ represents digital 1. 8-bit data transmission is shown in the Figure. The encoded data is '11011100'. One of the disadvantages of PPM is that the receiver must be synchronized with the sender.
Frequency Shit Keying is another modulation technique that it could be used with the Li-Fi. The idea behind this modulation technique is to use different frequencies for the logic 1 and 0 . An example on the generated modulated data is shown in Figure 6.

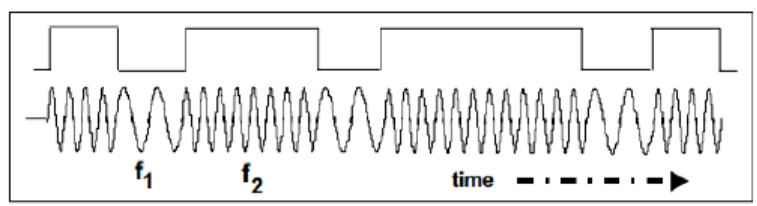

Fig. 6. Frequency shift keying example

Orthogonal Frequency Division Multiplexing (OFDM) is another but complex modulation technique that could be used with Li-Fi system. OFDM is considered as a multi-symbol modulation or multi carrier modulation. Different carriers with different frequencies that are orthogonal to each other. The block diagram for OFDM is depicted in Figure 7.

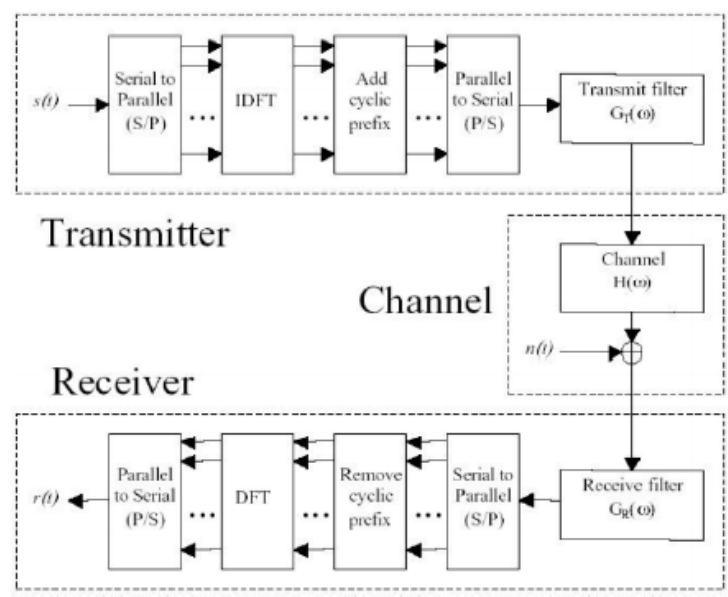

Fig. 7. OFDM block diagram

As can be seen in the Figure, the data is transferred from serial to parallel. This parallel data is used as an input to the Inverse discrete Fourier Transform (IDFT). The IDFT is applied to generate the orthogonal subcarriers waveforms. The block denoted as "Add Cyclic Prefix" is the most common guard interval (GI). The GI is added to eliminate the inter block interference (IBI). The cyclic prefix (CP) is a good substitute of the zero-padding GI. The receiver is the inverse of the transmitter operations. A simplified version of the OFDM in the Li-Fi system is shown in Figure 8.

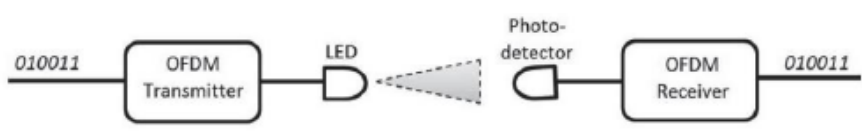

Fig. 8. OFDM for LI-FI [8]

One of the OFDM variations is introduced by [11] as indicated in Figure 9. The main idea is to use Delta Segma modulator 
after the cyclic prefix folowed by digital-to-analog converter (DAC). The generated signals are biased using 2 levels. Again the anvirs of these processes are utalized.

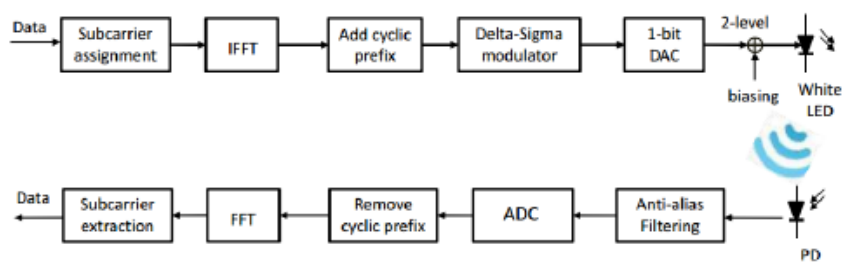

Fig. 9. Delta-Sigma OFDM

SIM OFDM is another variation of OFDM. The SIM OFDM technique splits the serial bit-stream into two bit-substreams of the same length. In this generic case, the original bit-stream is divided into three portions: a first bit-substream, a second bitsubstream, and a third bit-substream as shown in Figure 10 [1].

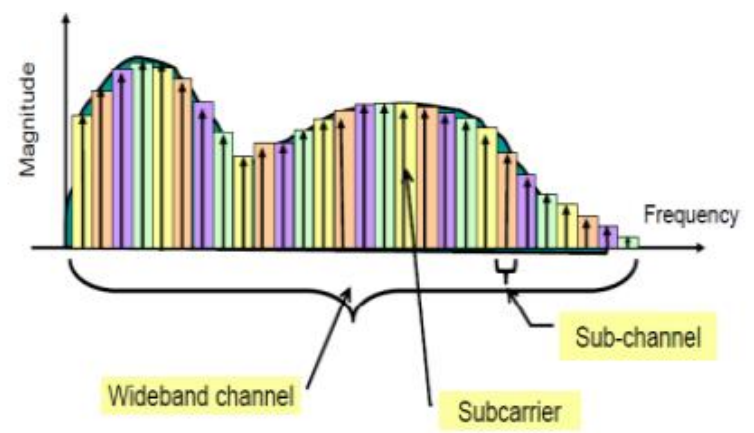

Fig. 10. SIM OFDM concept

\section{IS IT POSSIBLE FOR LI-FI TO REPLACE WI-FI TECHNOLOGY?}

Before answering the question of "Is it possible for Li-Fi to replace Wi-Fi?", let us specify the advantages and disadvantages of the Li-Fi.

\section{A. Li-Fi Advantages}

Clean Transmission media: Li-Fi uses light which is a clean transmission media that has none of the interferences.

Availability: The light is available everywhere and it is already used in houses, trains, vehicles, airplanes, and even in tunnels.

Security: Li-Fi signals are limited to the surrounding environment; therefore, the eavesdropping and other attacks are almost impossible.
Capacity: the visible light spectrum is 10,000 more than WiFi. High Li-Fi data rate can be achieved due to the low interference.

Efficiency: Li-Fi is distinguished by low cost even lower than the radio frequency technology. it is also can be used underwater in which it is one of the RF challenges.

\section{B. Li-Fi Disadvantages}

- Li-Fi signals are limited in range and incapable to penetrate solid surfaces, so the alignment between the transmitter and receiver are needed.

- Interference from other light sources could be a problem.

- Building a coverage network could be a challenge in the sense of connecting the transmitters and receivers.

- Reliability and availability especially during the day light could be another challenge.

- The technology still new and there is any type of standard.

\section{Potential Protoypes}

Because Li-Fi still new technology, almost only two prototypes are developed. One of this prototypes is developed by a company named PureLiFi [7] which it is a spin-out company by the University of Edinburgh. They are currently developing two prototypes; one is called Li-1st and the second is the Li-Flame. The Li-1st is just a simple platform for developing a Li-Fi system as shown in Figure 11. Where the Li-Flam is demonstrated at Mobile World Congress in Barcelona at the Scottish Development International stand. It consists of two components which are Li-Flame Ceiling Unit (CU) and Li-Flame Desktop Unit (DU) as shown in Figure 12 and 13. The main idea behind the Li-Flame is expalined through Figure 14.

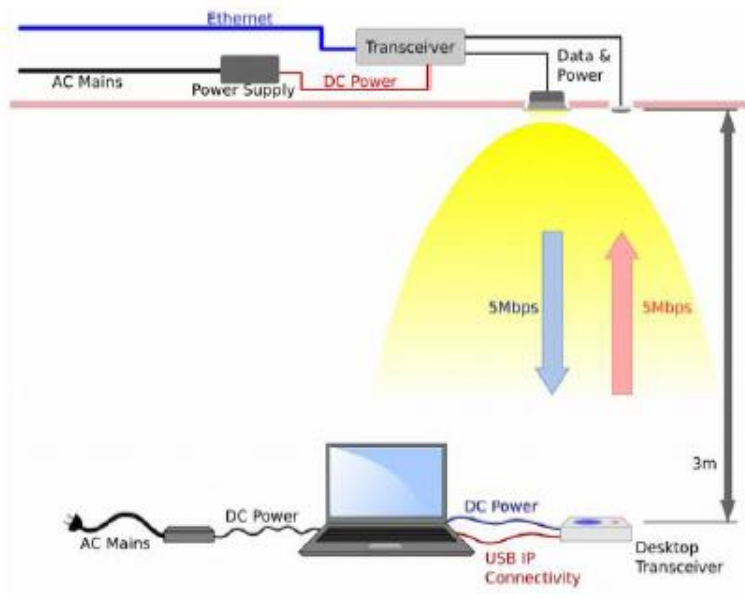

Fig. 11. Li-Fi 1st diagram 


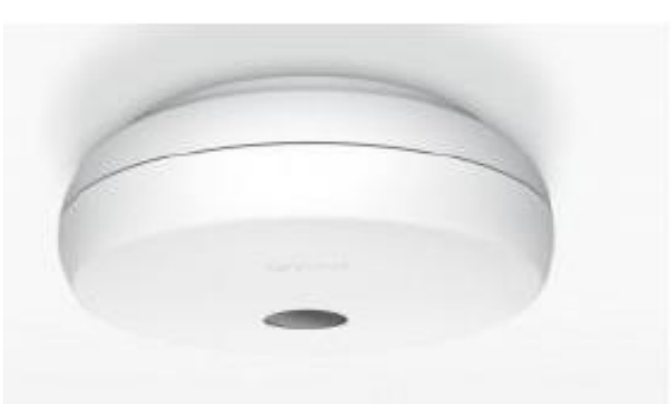

Fig. 12. Li-Flam Ceiling Unit (CU)

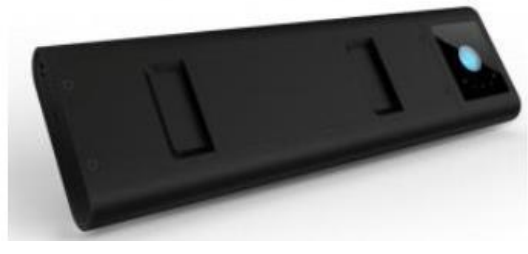

Fig. 13. Li-Flam Desktop Unit (DU)

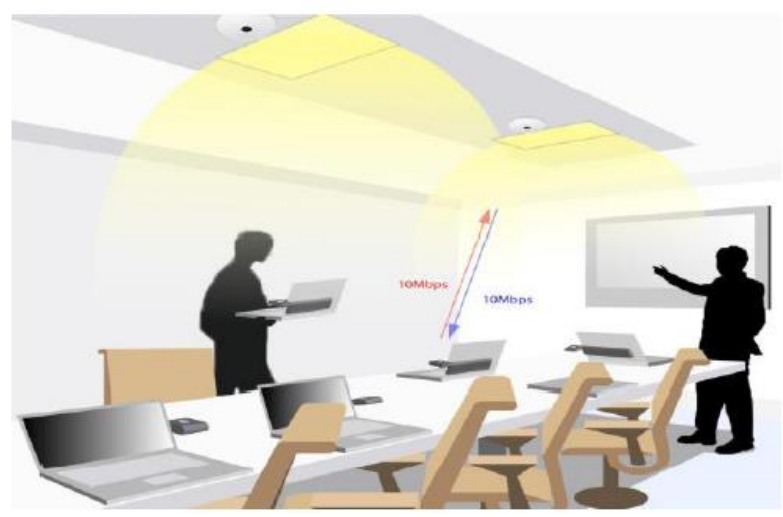

Fig. 14. Li-Flam system

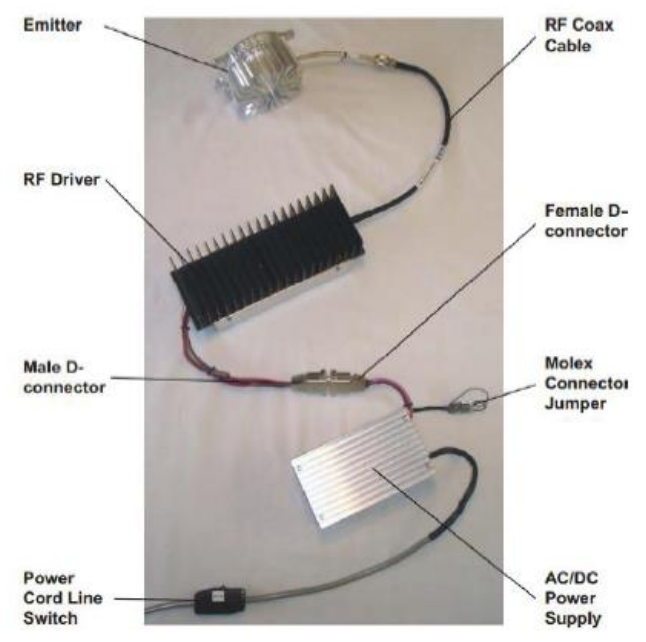

Fig. 15. AN016 KIT [5]
As can be seen in Figure 14, the CU will be hanged at the ceiling of the room and the DU will be connected to the computer. Expected data transfer rate is Mbps.

Another very simple prototype is produced by a company called LUXIM [5] entitled AN016 shown in Figure 15. AN016 is a very simple Kit that can be used in student class in which RF driver is utilized before the emitter LED.

\section{CONCLUSION}

As can be seen from the story of Li-Fi technology and effort, it seems that Li-Fi will have a great impact on the wireless technology. We believe that within few years, there will be a great advances to the Li-Fi technology. We are sure that once few prototypes to the Li-Fi systems are commercialized, the research in this field will take faster steps towards standardizing it. Therefore, our expectation is that Li-Fi will be the normal replacement to Wi-Fi within the next few years.

\section{REFERENCES}

[1]. Anurag Sarkar, Shalabh Agarwal, and Asoke Nath, "Li-Fi Technology: Data Transmission through Visible Light, "International Journal of Advance Research in Computer Science and Management Studies, Vol. 3, Issue 6, June 2015.

[2]. Emona Instruments Pty Ltd, "FSK - FSK -- FREQUENCY SHIFT FREQUENCY SHIFT KEYING , " can be found online at http://www.ele.uri.edu/courses/ele436/labs/newhandouts_09/D107_FSK-frequency_shift_keying.pdf, accessed on August 15, 2015.

[3]. G. Lazaridis , Pulse Position Modulation and Differential PPM , could be found online at , http://www.pcbheaven.com/wikipages/Pulse_Position_Modulation /, 2011, accessed on August 15, 2015.

[4]. Hass talk at TED, "'Wireless data from every light bulb," could be found

https://www.ted.com/talks/harald_haas_wireless_data_from_every _light_bulb, accessed on August 15, 2015.

[5]. Luxim, http://www.luxim.com/pdfs/demokitinstr.pdf

[6]. Matthew Wall, 'Li-fi' via LED light bulb data speed breakthrough," BBC News, 2013 , can be accessed online at , “ http://www.bbc.com/news/technology-24711935

[7]. Purelifi, http://purelifi.com/

[8]. R. Ndjiongue , H. C. Ferreira and T. M. N. Ngatched , "Visible Light Communications (VLC) Technology, "Wiley Encyclopedia of Electrical and Electronics Engineering 06/2015.

[9]. R. Madhura M , Ghdigaonkar Amit H., and Thara Rafat A. , “ DATA TRANSMISSION THROUGH VISIBLE LIGHT," International Journal of Electronics, Communication \& Instrumentation Engineering Research and Development (IJECIERD) ISSN 2249-684X Vol. 2 Issue 4 Dec 2012 81-88

[10]. Y. Xiao, "ORTHOGONAL FREQUENCY DIVISION MULTIPLEXING MODULATION AND INTER-CARRIER INTERFERENCE CANCELLATION “ Master Thesis, 2003, can be accessed online at http://etd.lsu.edu/docs/available/etd0411103-120728/unrestricted/Xiao_thesis.pdf

[11]. Y. Zhenhua, A. Redfern, and G. Tong Zhou, "Using Delta-Sigma Modulators in Visible Light OFDM Systems, " Proc. IEEE Wireless and Optical Communication Conference, Newark, New Jersey, 2014. 